\title{
The representational framework in perceptual theory
}

\author{
WILLIAM EPSTEIN \\ University of Wisconsin, Madison, Wisconsin
}

\begin{abstract}
The representational stance in perceptual theory is described and its proper domain of application is examined. A decision rule for invoking representation is proposed but, although the rule is principled, its implementation is problematic. Despite the widespread appeal of the representational stance, detailed working out of the position within perceptual theory is still needed.
\end{abstract}

Theoretical formulations of perception tend to be faithful to tradition. Notwithstanding the avowedly iconoclastic stance of ecological realism (Gibson, 1966, 1979; Michaels \& Carello, 1981; Reed \& Jones, 1982), contemporary theoretical conceptions exhibit striking continuities with traditions whose origins are in the philosophies of much earlier times. The hands of Descartes, Locke, and Berkeley are visible everywhere. In this essay, I propose to examine one of these traditions. Philosophers know the set of ideas under consideration as the representational theory of mind. The representational theory in philosophy treats the visual experience as the object of perception. That is, the object of perception is a representation of the world, or a "sense datum," for example, that in some sense resembles the world.

From the outset, the representational theory has had its dissenters among philosophers and has been the object of direct assault by a prominent 18 th century lexicographer. More recently, vigorous criticism has been voiced by philosophers in the Oxford network (e.g., Austin, Ayer, and Ryle). The controversial status of the representational theory of mind among philosophers has not inhibited scientific investigators of perception from importing the general framework into the theory of perception. The following is a self-description, volunteered by a thoroughly modern computational theorist:

From a philosophical point of view, the approach I describe is an extension of what have sometimes been called representational theories of mind. . . . Modern representational theories conceive of the mind as having access to systems of internal representations, mental states are characterized by asserting what the internal representations currently specify, and mental processes by how such internal representations are obtained and how they interact. (Marr, 1982, p. 6)

The Marr program is the development of a vision system

Address correspondence to W. Epstein, W. J. Brogden Psychology Bldg., University of Wisconsin, Madison, WI 53706. consisting of a hierarchical succession of levels of symbolic representation. The early representations are constrained by what is possible to compute from the retinal image, while higher levels are shaped by the information required to support adaptive behavior. The intermediatelevel representations are constrained by the information made explicit in the earlier representations and by the requirements of the succeeding ones.

The longevity of the representational framework is assured by its transmission to each new generation of novitiates. Introductory textbooks are for the most part made up of what the community elders judge to be unimpeachable, and for this reason these texts are useful guides to consensual wisdom. The following example illustrates how secure the representational theory is in the minds of contemporary experimental psychologists:

\begin{abstract}
A moment's reflection shows that there must be symbols inside our heads for the things we see, symbols which themselves are unlike the things they represent. Upon opening up a patient's head for a brain operation, the surgeon does not find there a miniature stage-set of the world! All he finds is a pink blancmange-like mass of brain cells. So it is an inescapable conclusion that there must be a symbolic description in the brain of the outside world, a description cast in symbols which stand for the various aspects of the world of which sight makes us aware.

The idea of visual experience as a symbolic process may seem a strange one. The likely reason for this is that the world we see-the visual world-is so very clearly "out there" that it can come as something of a shock to realize that somehow the whole of this world is tucked away in our skulls as an inner representation which stands for the real outside world. (Frisby, 1980, p. 9)
\end{abstract}

The present essay will evaluate the place of representations in accounting for perception. The dominant positions in the current theoretical landscape are occupied by the complete representationalist who sees representations at work at every juncture and the determined skeptic who considers representationalism to be a bit of antiquated mischief. My principal aim is to argue for a third position, 
a tertium quid, which reserves an important role for representation while denying it the all-encompassing role assigned to it by the perfect believer. Before turning to this matter, I will comment on the nature of representation, particularly on the difference between representations and mappings, and on the work that has been traditionally assigned to representations in theoretical formulations of perception.

\section{What is a Representation?}

A representation, according to the preceding excerpt, is something that stands for something else. To stand for a represented domain, the representation does not have to be a re-presentation. The representations that are active in theoretical formulations of the perceptual process are not iconic images of the represented domain. Rather, a representation typically is taken to be a symbolic recoding that preserves the information about objects and relations in the represented domain.

There is good agreement among advocates of the representational framework (see Fodor, 1975; Marr, 1982; Palmer, 1978; Pylyshyn, 1983; Rumelhart \& Norman, 1988) that the only useful way to talk about representation is in conjunction with processes that interpret the representation-processes that make the information in the representation explicit. "In all cases . . . it is necessary to recognize that a representational system is incomplete unless both the representation and the processes that operate upon them have been explicitly considered" (Rumelhart \& Norman, 1988, p. 9). And Palmer urges the same point even more strongly:

The relations in a representation are operational relations rather than apparent relations. Operational relations are simply those defined by the processes that interpret the representation. . . . Without these processes, the representation is meaningless. ... The only information contained in a representation is that for which operations are defined to obtain it. (Palmer, 1978, pp. 266-267)

Consequently, although we may be tempted to talk about implicit and explicit information in a representation, this sort of talk is not coherent. Given a representation and an appropriate set of processes, this relational double defines information with nothing left over; if the appropriate processes for extracting information are not available, then that information is not contained in the representation either explicitly or implicitly.

Representations and mappings. The obligatory coupling of representation and the information-extraction process serves to highlight the difference between a mapping and a representation. All representations are instances of mapping but all mappings are not representations. A relationship between two domains will qualify as a mapping if the domains embody, in some form, the same objects and relations - that is, if there is good correspondence between the two domains. This requirement also must be satisfied by the relationship between the represented and representing domains. But as I have just noted, more than correspondence is required to qualify as a representation.
A process for making the objects and relations explicit must be postulated.

In the absence of the interpretive process, even an ideal mapping-a picture delivered to the mind-would not qualify as a representation. This counterintuitive assertion gains credibility when it is recalled that a representation is a symbolic recoding. An event can only assume symbolic status when it is assigned that status by an agent or process that is endowed with intentionality. That is, to be a symbol is to have a prescribed status in a rulegoverned symbolic system. The picture delivered to the mind would not qualify in the absence of a process that could explicate the symbolic network of pictorial representation.

At the cost of preaching to the converted, I will make one more pass at the mapping-representation distinction. A relationship between domains secures status as a mapping solely by virtue of correspondence, irrespective of any assigned functional significance. There is no obligation to exhibit the real or putative function of the mapping in the information-processing system. A representation, on the other hand, only secures its status when it can be located in a conception of part or whole of an information-processing system. A paradigm case is Marr's (1982) deployment of representation. The "primal sketch," for example, qualifies as a representation in the full sense intended here: It is a selective recoding that makes explicit properties and relations in the optical input in the services of an overall information-processing system. The functional theory provides the motivation and part of the justification for positing the primal sketch representation. In the absence of theory, both motivation and justification would be lacking. I think there is a cautionary homily here for neuroscientists who locate representations in every wrinkle in the brain.

Infinite regress. There is a ghost in the representational attic who is too clamorous to ignore. Interpretation, after all, is a process that issues in representations; the representations that comprise an interpretation are about the representations that are being interpreted and their relationship to their objects. If the representations that comprise an interpretation have to be interpreted themselves, there would be a regress of interpretive processes. The processes must stop somewhere and the final representation must itself be uninterpreted.

Either the representationalist must turn tail in the presence of the prospect of infinite regress or stand ground and admit uninterpreted representations. Neither response can be contemplated equably by the representationalist. Is there a way to escape the horns of this dilemma? One exit is gained by allowing that the ultimate product of the process of iterative representation is a nonsymbolic description expressed in an internal code, which is directly accessible to the visual system.

The model for this argument is the distinction between the input-output language and the machine language of computers. As the term implies, input-output language supports communication between the computer and the environment. Machine language is the language in which 
the computations are run. In order to use the input-output language, the machine must deploy a compiler that mediates between the two languages. But no compiler (mediator, interpreter) is required for the machine language. Fodor, in his defense of the "language of thought," puts the matter nicely:

What avoids an infinite regress of compilers is the fact that the machine is built to use the machine language. Roughly, the machine language ... formulae correspond directly in computationally relevant physical states and operations of the machine: The physics of the machine thus guarantees that the sequences of states and operations it runs through ... respect the semantic constraints on formulae in its internal language. What takes the place of a truth definition for the machine language is simply the engineering principles which guarantee this correspondence. (Fodor, 1975 , p. 66)

There are variants on this theme (e.g., Haugeland, 1978; Pylyshyn, 1989). The common core is neatly summarized by Hatfield (1991): "Appeal is made to causal properties of symbols that do not depend on their being 'read' as symbols, but that depend instead upon their brute physical properties and the physical structure of the entity (device or organism) in which they are instantiated" (p. 166).

Applying this model to the human visual system, the dreaded infinite regress is averted by supposing that the representational system is grounded in a physical system in which interactions occur not in virtue of interpretations of symbols and rules governing interactions, but in virtue of the design of the system and its components. Causal effectiveness at this level does not depend on reading of representations; indeed, it is a category error to predicate reading or representation to the system at this level. The grounding system is what it is and its activity is an instantiation of what it is.

\section{THE WORK THAT REPRESENTATIONS DO}

The representational framework may have its roots in centuries-old opinion, but its contemporary status cannot be explained by reference to historical precedent. The current vitality of the representational framework must be due to the work that it does in the theory of perception.

\section{Getting the Outside Inside}

Positing representations is a way of reconciling a sharp disparity between the phenomenology of everyday seeing and the scientific analysis of the possibilities of seeing. The experience of ordinary seeing is one of direct contact with the world. But, so the argument goes, even cursory analysis shows that all that is directly available to the percipient is the light reflected from surfaces in the world onto receptive surfaces of the eye. How can this fundamental fact be reconciled with the nature of the experience of seeing? And, moreover, how can the fact that only light gets in be reconciled with the fact that it is the world that we see-not light? (Indeed, what could it mean to say that we see light?) Both questions are resolved by the introduction of representations. It is representations that are experienced directly, and because the representations reproduce the information in the represented world, the experience of perception is one of direct contact with the world. In this way, representations get the outside inside.

I think that considerations of this sort do inspire representationalism (e.g., see previous excerpt from Frisby, 1980). Nevertheless, there are very serious difficulties with this line of thinking. As one example, if direct perception is only of representations, how does the percipient come to know what external objects are like, or which representations they resemble? Since we can only perceive representations, whenever we may think that we are observing external objects to compare them with representations or to discover their intrinsic nature, we are only observing other representations. In general, it is difficult to discover an escape from the world of representations.

Criticisms of the sort described above are not novel (e.g., for thorough treatment see Hirst, 1959; Katz, 1983; and Ryle, 1949 and the writings of the ecological realists, e.g., Mace, 1977). I am not going to rehearse and evaluate these arguments because I think that the appeal of representationalism in contemporary accounts of perception is to be found elsewhere. Moreover, although I am not satisfied with the efforts that representationalists have made to dissolve the problems of the representational theory of mind, I am not persuaded, as are some critics (e.g., Hacker, 1987), that failure to resolve these conundrums robs the representationalist framework of any utility. A scientific paradigm may have great inclusive utility despite ambiguity or seeming paradox in important matters.

\section{Inference, Computation, and Representation}

The dominant conception of the perceptual process may be labeled constructivism. The perceptual world is constructed or assembled from the raw material of sensory input and stored knowledge. The process of construction has been likened to inference or problem solving and more recently the process has been characterized as computational. The representational framework serves as a superstructure for support of this conception of the perceptual process. Proponents of the computational/representational approach argue that the only plausible story of perception is computational and the only plausible computational story must assume a representational system in which the computations are executed.

It is undeniable that if a variant of the constructivist/ computational approach is adopted then the representational framework is needed to allow the approach to proceed smoothly. Any theory that postulates a process resembling nondemonstrative inference (e.g., Gregory, 1966; Rock, 1983) or a process of successive computations (e.g., Marr, 1982) has need to postulate a representational medium for the display of "premises" or the display of representations-that is, the output of processes (algorithms) that operate over mappings. No one has been more straightforward and exacting in promoting this ap- 
proach than Marr. Other advocates of the computational/ representational approach have been content to offer general formulations. In sharp contrast, Marr has set out an account at a level of explicitness not even approximated by his predecessors. Moreover, it is typically the case for more traditional constructivist accounts that it is difficult to say just what observations would stand as affirmation of the account-that is, what observations would establish that the putative representations play a causal role. We are asked to accept arguments from analogy or arguments based on selective refutation. In Marr's case, an objective, although somewhat problematic procedure is deployed. The test of the reality of the imputed representations is implementation of a successful procedure-a computer program that will deliver the desired solution.

These remarks concerning Marr's (1982) project point to one of the principal sources of encouragement for representationalism. Marr's allegiance to representationalism owes much to what is old-the representational theory of mind-but he is equally affected by the very new in the form of contemporary work in machine vision. The machine vision enterprise is inherently representational. Marr makes no secret that he is inspirited by the computer metaphor.

It is important to recognize the extent of Marr's dependence. Consider that although Marr's is a vision of an information-processing system inhabited by representations, nowhere does he attempt to adduce evidence that representations are in fact generated and organized in the human visual system in the prescribed manner. Instead, Marr relies on the formal proof of computer implementation and the appeal to intuition that what is actual in a computer is possible (likely?) in a human.

There are many reasons to be wary of this form of argument. For example, the human visual system has been designed by the forces that drive evolution, while the design of the machine vision system has been shaped by other constraints. There is no need to rehearse the familiar arguments concerning the potential risks and benefits of the developing symbiosis involving machine vision and human vision. It suffices for my purpose to mark the influence of the machine vision model, and to recognize that while computer models may augment the plausibility of analogous accounts of human vision, they cannot stand in for the direct evidence that is wanted.

\section{Perception of Nonprojectable Properties}

The representational framework has secured the status of "normal" science, no longer requiring detailed justification. So it is good to have available the work of two astute analysts of the theoretical foundations of psychology who have taken on the task of explicating the presuppositions and promise of the representational framework. Fodor $(1975,1981)$ and Pylyshyn (1983) will reward the diligent reader. What follows was stimulated by reading the answers that these two representationalists offer to the central question of this section, but my treatment should not be attributed to these writers.
Development of the answer begins with a distinction between two types of properties of objects of perception: projectable and nonprojectable properties. The former are properties of the environment that are nomically related to properties of light for which there are known or plausible candidate transducers in the visual system. Spatial layout and motion are two examples. Nonprojectable objects of perception are properties of the environment that do not satisfy these two requirements: as far as we know, these properties neither are captured by information in stimulation, nor do we know of a candidate transducer. The reason that many objects of perception are nonprojectable is because it seems implausible to suppose that for these perceptual categories there is a finite physical description that is invariant over the set of objects that constitute the membership of the category.

When the possibility of such physical description is ruled out in principle-that is, when there is no prospect of identifying a nomic relationship between distal and proximal properties (one underwritten by physical law), then there is no reason to suppose that a transducer has evolved in an actual biological system to support perception of the distal property. On the other hand, when the requirement of nomic relationship is satisfied and the distal property is demonstrably important to the adaptive success of the species, then evolutionary design of a transducer is a plausible assumption. The former circumstances apply in the case of nonprojectable properties; the latter apply in the case of projectable properties.

With this division in mind, we are ready to consider the work that representations do. Representations are required in order to explain the ability of many biological vision systems to respond selectively to nonprojectable properties of objects. It is the need to account for nonprojectable properties that motivates the move toward representations. There seems little need to invoke representations for the perception of projectable properties. When optical structures that specify properties and events of the world are available and when the visual system has evolved mechanisms to register these spatiotemporal optical structures, the demands of an effective visual system are satisfied without invoking computations over representations. When little is enough, more is too much, and invoking representations is too much when pick-up of information by specially designed mechanisms will suffice. $^{1}$

Understanding perception of nonprojectable properties, on the other hand, cannot be achieved without stipulating operations over representations. Indeed, archaeologists of the mind who find inspiration in the power of the evolution epic might speculate that the adaptive utility of the perception of nonprojectable properties fostered selection for gene pools that supported brain structures with representational capabilities.

It is important to understand that an object of perception often will have both projectable and nonprojectable properties. Consider environmental structures that afford containment such as boxes, tins, or bottles, and suppose 
that containment is perceived. Inasmuch as all of these structures are physical structures, it must be the case that there will be many properties of these structures that are projectable-that is, these properties will be related nomically to optical structures and it seems reasonable to suppose that candidate transducers will be at hand. But the nonprojectable property, "affording containment," is not a physical property of this sort; neither nomic relationship nor transducer is apparent in virtue of the property containment.

To summarize, the perceptual world is constituted of more than the class of properties that are picked out by the standard dimensions of physics. The Gestalt theorists (see Koffka, 1935, chap. 8) recognized this; Gibson (1979) in his radical ontology emphasized this point and, just as importantly, ordinary experience is unequivocal on this matter. We do see "edibility," "shoeness," and "sit-upon-ableness" and so much the worse for the critic who insists that we cannot do so. Moreover, perception of properties of this sort is vitally implicated in the support of adaptive behavior. To understand this capability, a representational framework is essential. The perception of nonprojectable properties requires a process resembling concept formation, a computational process that runs in a representational medium. No perception of nonprojectable properties without representations. ${ }^{2}$

The foregoing argument has the ring of the new, but I can't help wondering whether I have heard this song before. The following familiar refrain comes to mind: The relationship between distal environmental structures and optical structures is such that optical input commonly is informationally impoverished, offering no prospect of linking perception directly to information. An inferencelike process must be invoked to explain why the world looks as it does. Inferences call for the presentation of premises and the evaluation of solutions and these operations presuppose a representational system.

Is the argument from the perception of nonprojectable properties fundamentally different than the older argument from informational impoverishment? The argument from nonprojectables rests on the premise that there are, in fact, adaptively significant properties of the environment that cannot be specified by information in stimulation. The informational shortfall is a shortfall in principle. But we ought to be wary of such claims. The history of the investigation of perception shows that these claims often reflect special construals of the nature of stimulation and information rather than claims that compel acceptance independently of starting point.

Consider the case of spatial layout. Berkeley's (1709) New Theory of Vision opens with an assertion of the intractable equivocality of optical input with respect to spatial layout. Berkeley believed that he was advancing an argument that was unimpeachable in principle. The ensuing 250 years gave no reason to question Berkeley's assessment. The principle of intractable equivocality assumed canonical status and was prominently displayed early in discussions of the perception of visual space. But owing initially to the insights of J. J. Gibson (1966), the contemporary assessment is quite different. We have come to recognize that the claim of intractable equivocality is not irresistible. The seeming unimpeachability of the claim was due to adoption of a special stance regarding stimulation and information. Once this stance was modified, the claim lost all force; in fact, among investigators of the perception of spatial layout, the dominant view is that optical input is informationally rich. Spatial layout has been transformed from a nonprojectable property into a projectable property.

A representationalist might cede this particular argument but not surrender the general argument. Admittedly, the assignment of spatial layout to the class of nonprojectables was an error, but the general thesis that many of the objects of perception are nonprojectable is not contradicted. Certainly there are perceptual categories for which there is no invariant physical description over the set of objects that constitute the membership of the category. Granted, it was a mistake to take perceived spatial layout to be one such perceptual category, but there is an ample supply of other candidates nevertheless.

The case of spatial layout should cause us to pause. If a new stance regarding the information for perception led to a reassessment of the status of spatial layout, can we confidently rule out similar shifts for other properties that presently are considered nonprojectable? The question is not merely rhetorical. One way of reading Gibson's (1979) program and the ecological realists is that the aim is mass reallocation of perceptual properties from the nonprojectable into the projectable category. A number of modest successes have been reported. Consider a set of stairs. Typically we see directly that the stairs do or do not allow normal, upright bipedal ascent. How is this determination made? On the basis of their discussion of the determination of a related functional property, here is how two eminent representationalists (Rumelhart \& Norman, 1988 , p. 71) would respond. Mental simulations are executed in a representational medium. A mental model of the stair structure is formed and ascent is simulated. Various simulations are run and their outcomes determine the person's determination of climbability. But a very different answer has been developed and evaluated experimentally by Warren (1984). According to Warren, there is optical information to specify the relationship of stair riser height to leg length - between the environmental property and the relevant property of the observer's action system-and this information picks out the perceptual category "climbable" without recourse to computation in a representational medium.

Once we are rid of presuppositions concerning the possibilities of informational specificity, the division between projectable and nonprojectable properties must be drawn cautiously. Nevertheless, there are properties that seem to be convincing candidates for membership in the class of nonprojectables. Many functional properties that owe their status to cultural convention seem to fit the bill. Take the property "mailbox." Setting out to post a letter in 
an unfamiliar country serves to remind one that the property of affording letter-posting is culture-specific. The function of the red cylindric object found on London streets is not obvious to the stranger.

Other nonprojectable property candidates are those that owe their status to experience that is context-specific. For example, consider the proprietary relationship. Seeing that the chair is "Lila's chair" is as immediately accessible and effortless as seeing the size and color of the chair. But it is clear that the property "Lila's chair" is not a projectable property. The proprietary relationship is established by recurrent exposure to transactions involving Lila and the chair. The proprietary relationship is not specified by information in occurrent optical structures, nor is the proprietary relationship the sort of property for which it is reasonable to posit a transducer designed by evolution for encoding this property.

What these properties and others like them have in common is that they are the properties they are in virtue of access to special knowledge or experience. To explain the fact that the Londoner sees a postal box, whereas I see a red cylinder of unknown function and that I see Lila's chair and you see merely a chair, there is no recourse but to turn to the shared knowledge of Londoners that is not available to visitors and to the accumulated experience of transactions between a particular person and a particular chair. It is hard to know how to tell a story about these perceptual attainments that does not rely heavily on the representational framework.

\section{Overview}

I take the view that the representational framework is needed to explain perception of nonprojectables, but that it is a mistake to rush to classify properties as nonprojectable. Still, I have agreed above that there are nonprojectable properties that are perceived. And I also agree that an account of these perceptual categories requires recourse to representations. But the representationalist accounts I know are typically sketchy and unconvincing. I think that it is time the representationalists began taking themselves seriously.

\section{REFERENCES}

BERKELEY, G. (1709). An essay toward a new theory of vision. London: J. M. Dent \& Sons.

FoDOR, J. (1975). The language of thought. New York: Thomas Y. Crowell.

FoDOR, J. (1981). Representations. Cambridge, MA: MIT Press.

FrisBy, J. (1980). Illusion, brain and mind. Oxford: Oxford University Press.

GrBson, J. J. (1966). The senses considered as perceptual systems. Boston: Houghton Mifflin.

GrBson, J. J. (1979). The ecological approach to visual perception. Boston: Houghton Mifflin.

GREGORY, R. (1966). Eye and brain. New York: McGraw-Hill.

HACKER, P. (1987). Appearance and reality. Oxford: Blackwell.

HatField, G. (1991). Representation in perception and cognition: Connectionist affordances. In W. Ramsey, S. P. Stich, \& D. E. Rumelhart (Eds.), Philosophy and connectionist theory (pp. 163-195). Hillsdale, NJ: Erlbaum.

HaUgeland, J. (1978). The nature and plausibility of cognitivism. Behavioral \& Brain Sciences, 1, 215-226.
Hirst, R. J. (1959). The problems of perception. London: George Allen \& Unwin.

KATZ, S. (1983). R. L. Gregory and others: The wrong picture of the picture theory of perception. Perception, 12, 269-279.

KoFFKA, K. (1935). Principles of Gestalt psychology. Boston: Harcourt, Brace.

MACE, W. (1977). James Gibson's strategy for perceiving: Ask not what's inside your head, but what your head is inside of. In $\mathbf{R}$. Shaw \& J. Bransford (Eds.), Perceiving, acting and knowing (pp. 43-65). Hillsdale, NJ: Erlbaum.

MarR, D. (1982). Vision. New York: W. H. Freeman.

Michaels, C. F., Carello, C. (1981). Direct perception. Englewood Cliffs, NJ: Prentice-Hall.

PALMER, S. E. (1978). Fundamental aspects of cognitive representation. In E. Rosch \& B. B. Lloyd (Eds.), Cognition and categorization (pp. 259-303). Hillsdale, NJ: Erlbaum.

Pylyshyn, X. (1983). Computation and cognition. Cambridge, MA: MIT Press.

Prlyshyn, X. (1989). Computing in cognitive science. In M. I. Posner (Ed.), Foundations of cognitive science (pp. 49-92). Cambridge, MA: MIT Press.

REED, E., \& JONES, R. (Eds.). (1982). Reasons for realism. Hillsdale, NJ: Erlbaum.

Rock, I. (1983). The logic of perception. Cambridge, MA: MIT Press. Rumelhart, D., \& Norman, D. (1988). Representation in memory. In R. C. Atkinson, R. J. Herrnstein, G. Lindzey, \& R. Duncan Luce (Eds.), Stevens' handbook of experimental psychology (pp. 511-587). New York: Wiley.

RYLE, G. (1949). The concept of mind. New York: Barnes \& Noble. WARREN, W. (1984). Perceiving affordances: Visual guidance of stair climbing. Journal of Experimental Psychology: Human Perception \& Performance, 10, 683-703.

\section{NOTES}

1. Earlier I offered Marr's primal sketch as an exemplary instance of representation that highlights the difference between a mapping and a representation and that exhibits the need to talk about representations in tandem with a process. If, as is true for Marr, one adopts the representational stance without qualification, then Marr is a fine model. But I am proposing to adopt a middle position on this matter. Accordingly, I do not endorse Marr's unreserved commitment to representationalism. When Mart is concerned with analysis or perception of projectable properties, such as 3-D structure and motion, the attributions to representations and representational transformation may be questioned. As I have commented earlier, Marr's commitment was informed by the style of analysis and modeling that dominated the computer vision enterprise in the 1970 s. No independent argument for representational transformation based on analysis of the human visual system is developed by Marr. Recent parallel developments in conceptions of brain process, computer architecture, and cognitive science make these disciplines less reliable as partners in the representational enterprise.

2. One reviewer suggested that the distinction I draw between perception of projectable and nonprojectable properties might be better drawn between perception and cognition. For a number of reasons, I decline to make this move. First, adopting the suggestion incurs the risk of obscuring the phenomenological facts. When perceptual experience is consulted, we do not mark a difference between perceiving a nonprojectable and perceiving a projectable property. The perceptioncognition dichotomous classification tends to disguise this important fact. Also, the distinction between perception of projectable and nonprojectable properties sets out to be a principled distinction. By contrast, a settled distinction between perception and cognition has been elusive. Admittedly, in both cases, the boundaries cannot be fixed permanently, but I would argue that in the former instance the boundary shifts will reflect knowledge, for example, new understanding of the properties of spatiotemporal structures. In the latter case, boundary shifts will likely follow changing fashions in conceptual dress.

(Manuscript received August 3, 1992; revision accepted for publication November 24, 1992.) 